\title{
The giant radio halo in Abell 2163
}

\author{
L. Feretti ${ }^{1}$, R. Fusco-Femiano ${ }^{2}$, G. Giovannini ${ }^{1,3}$, and F. Govoni ${ }^{1,4}$ \\ 1 Istituto di Radioastronomia - CNR, via P. Gobetti 101, 40129 Bologna, Italy \\ 2 Istituto di Astrofisica Spaziale CNR, via del Fosso del Cavaliere, 00133 Roma, Italy \\ 3 Dipartimento di Fisica, Univ. Bologna, Via B. Pichat 6/2, 40127 Bologna, Italy \\ 4 Dipartimento di Astronomia, Univ. Bologna, via Ranzani 1, 40127 Bologna, Italy
}

Received 7 February 2001 / Accepted 11 April 2001

\begin{abstract}
New radio data is presented for the rich cluster Abell 2163. The cluster radio emission is characterized by the presence of a radio halo, which is one of the most powerful and extended halos known so far. In the NE peripheral cluster region, we also detect diffuse elongated emission, which we classify as a cluster relic. The cluster A2163 is very hot and luminous in X-ray. Its central region is probably in a highly non relaxed state, suggesting that this cluster is likely to be a recent merger. The existence of a radio halo in this cluster confirms that halos are associated with hot massive clusters, and confirms the connection between radio halos and cluster merger processes. The comparison between the radio emission of the halo and the cluster X-ray emission shows a close structural similarity. A power law correlation is found between the radio and X-ray brightness, with index $=0.64$. We also report the upper limit to the hard X-ray emission, obtained from a BeppoSAX observation. We discuss the implications of our results.
\end{abstract}

Key words. radio continuum: general - galaxies: clusters: general - galaxies: clusters: individual: A2163 intergalactic medium - X-rays: galaxies: clusters

\section{Introduction}

Recent observations of clusters of galaxies have revealed a new and complex scenario in the structure of the intergalactic medium. The clusters are not simple relaxed structures, but are still forming at the present epoch. Substructures, commonly observed in the X-ray distribution of a high number of rich clusters (Henry \& Briel 1993; Burns et al. 1994), are evidence of the hierarchic growth of clusters from the merger of poorer subclusters.

An important problem in cluster phenomenology involves cluster-wide radio halos, whose prototype is Coma C (Giovannini et al. 1993, and references therein). These are extended diffuse radio sources located at the cluster centers, with typical sizes of $\gtrsim 1 h_{50}^{-1} \mathrm{Mpc}$, regular shape, steep radio spectra and no significant polarization. Diffuse radio sources, named relics, have also been detected in peripheral regions of the clusters. They are irregular in shape and generally highly polarized. The origin and properties of halos and relics are still being debated. According to recent suggestions, the cluster merger process may play a crucial role in the formation and energetics of these sources (see Feretti \& Giovannini 1996 and references therein, Feretti 1999).

Send offprint requests to: L. Feretti, e-mail: lferetti@ira.bo.cnr.it
Abell 2163 is a distant $(z=0.203$, Struble \& Rood 1999), rich cluster, and is one of the hottest (mean $k T=$ 12-15 keV, Elbaz et al. 1995; Markevitch et al. 1996), and most X-ray luminous $\left(L_{\mathrm{X}[2-10 \mathrm{keV}]}=6 \times 10^{45} \mathrm{erg} \mathrm{s}^{-1}\right.$, Arnaud et al. 1992) among known clusters. A BeppoSAX observation measures a temperature of $10-11 \mathrm{keV}$ out to $4^{\prime}$, with a marginally significant $(<2 \sigma)$ rise in temperature at larger radii (Irwin \& Bregman 2000). According to the morphological study of Elbaz et al. (1995), based on ROSAT data, and the spectroscopic analysis of Markevitch et al. (1996), based on ASCA data, the cluster is likely to be a recent merger. A highly nonrelaxed state in the cluster inner region is confirmed by the recent Chandra results (Markevitch et al. 2000). A strong Sunyaev-Zel'dovich (SZ) effect has been reported in this cluster (Holzapfel et al. 1997; Désert et al. 1998).

We present here the radio data of A2163 obtained with the Very Large Array (VLA). In particular, we analyze in detail the diffuse radio emission whose presence was reported by Herbig \& Birkinshaw (1994). We also give data for the extended radio galaxies. In addition, we report the results obtained by the Phoswich Detector System (PDS) on board BeppoSAX to search for hard X-ray radiation.

We adopt $H_{0}=50 \mathrm{~km} \mathrm{~s}^{-1} \mathrm{Mpc}^{-1}$ and $q_{0}=0.5$. With these values, 1 arcsec corresponds to $4.27 \mathrm{kpc}$ at the distance of A2163. 


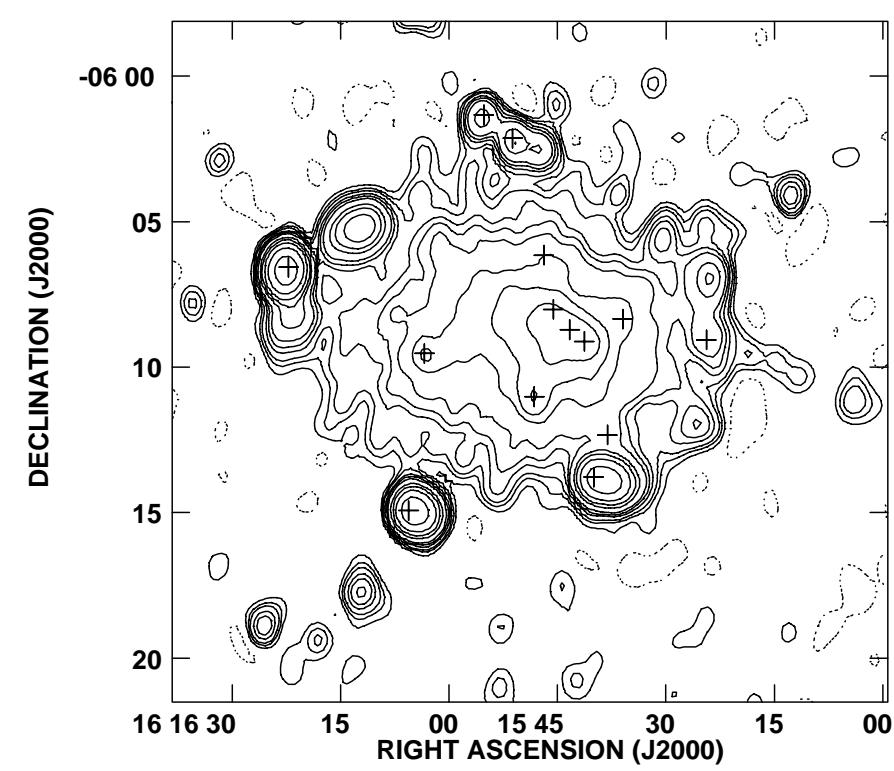

Fig. 1. Radio map at $20 \mathrm{~cm}$ with resolution of $45^{\prime \prime} \times 60^{\prime \prime}$ $(\mathrm{RA} \times \mathrm{DEC})$. The $\sigma$ noise level in this map is $0.03 \mathrm{mJy} / \mathrm{beam}$. Contours are at $-0.1,0.1,0.2,0.3,0.5,0.7,1,2,3,5,10$, $25 \mathrm{mJy} /$ beam. Crosses indicate the discrete radio sources detected from the higher resolution images.

Table 1. Observing log.

\begin{tabular}{lllll}
\hline $\begin{array}{l}\text { Freq. } \\
\mathrm{MHz}\end{array}$ & $\begin{array}{l}\text { Bandwitdh } \\
\mathrm{MHz}\end{array}$ & Array & Date & $\begin{array}{l}\text { Duration } \\
\min \end{array}$ \\
\hline $1365 / 1465$ & 50 & $\mathrm{D}$ & 02 Feb. 1998 & 140 \\
$1365 / 1465$ & 25 & $\mathrm{C}$ & 02 Jan. 1999 & 200 \\
$1365 / 1465$ & 25 & $\mathrm{~A}$ & 21 May 1998 & 50 \\
$4885 / 4835$ & 50 & $\mathrm{D}$ & 26 Dec. 1997 & 120 \\
\hline
\end{tabular}

\section{Radio observations}

Radio observations were obtained with the VLA in different configurations (see Table 1) at $20 \mathrm{~cm}$, and with the $\mathrm{D}$ array at $6 \mathrm{~cm}$. The data were calibrated and reduced with the Astronomical Image Processing System (AIPS), following the standard procedure (Fourier inversion, CLEAN and RESTORE, selfcalibration).

At $20 \mathrm{~cm}$, images with angular resolutions ranging from $\sim 1.5^{\prime \prime}$ to $60^{\prime \prime}$ were produced. At the lowest resolution, maps for the two observing frequencies were produced separately, to get some information about the spectral index. We also obtained maps of the polarized intensity in the standard way. An image at $6 \mathrm{~cm}$ was produced with resolution $15^{\prime \prime} \times 20^{\prime \prime}(\mathrm{RA} \times \mathrm{DEC})$, and a rms noise level of $0.35 \mathrm{mJy} /$ beam.

\section{Results}

\subsection{Radio halo and other diffuse sources}

The radio image of this cluster is shown in Fig. 1, where the crosses mark discrete unrelated sources detected in
Table 2. Diffuse sources.

\begin{tabular}{lll}
\hline & Halo & Relic (D3) \\
\hline Flux density $-20 \mathrm{~cm}(\mathrm{mJy})$ & $155 \pm 2$ & $18.7 \pm 0.3$ \\
Largest size $(\mathrm{kpc})$ & $2900 \pm 100$ & $640 \pm 50$ \\
Spectral index & $1.6 \pm 0.3$ & $2.1 \pm 0.3$ \\
Power $-20 \mathrm{~cm}\left(\times 10^{24} \mathrm{~W} \mathrm{~Hz}^{-1}\right)$ & $30.1 \pm 0.4$ & $3.63 \pm 0.06$ \\
Luminosity $\left(\times 10^{35} \mathrm{~W}\right)$ & $13.6_{-7.8}^{+26.9}$ & $10.6_{-7.3}^{+26.6}$ \\
Volume $\left(\times 10^{7} \mathrm{kpc}^{3}\right)$ & $\sim 950$ & $\sim 3.2$ \\
$u_{\text {min }}\left(\times 10^{-14} \mathrm{erg} \mathrm{cm}^{-3}\right)$ & 8.2 & 36 \\
$U_{\text {min }}\left(\times 10^{61} \mathrm{erg}\right)$ & 2.3 & 0.34 \\
$H_{\text {eq }}(\mu \mathrm{G})$ & 0.9 & 2.0 \\
\hline
\end{tabular}

the map at $4^{\prime \prime}$ resolution, with a brightness higher than $0.2 \mathrm{mJy} /$ beam. The diffuse emission permeates the cluster center for a total extent of $\sim 11.5^{\prime}$, corresponding to $\sim 2.9 \mathrm{Mpc}$. With this size, the halo in A2163 is the largest known radio halo. It displays a very regular shape, slightly elongated in the E-W direction. The low brightness emission does not fade into the noise, but shows rather sharp boundaries. This is an indication that the whole structure is properly imaged here. We made polarization maps of the cluster at $20 \mathrm{~cm}$. Some polarized flux is detected at a level of $2-4 \%$. Because of the noise, this should be considered as an upper limit.

At higher angular resolution (Fig. 2), only the central region of the radio halo is detected. All the discrete sources embedded within the diffuse emission are easily visible. The high resolution image reveals the existence of diffuse features at the periphery of the cluster (labeled D1, D2, D3 and D4 in Fig. 2). The diffuse emission D1 seems to be coincident with a faint galaxy. The northern source D2 could be related to a nearby point-like source. The two emissions D3 and D4 do not show any obvious optical counterpart. If they were both associated with the strong eastern radio source at position $\mathrm{RA}=16^{\mathrm{h}} 16^{\mathrm{m}} 22.2^{\mathrm{s}}$, $\mathrm{DEC}=-06^{\circ} 06^{\prime} 34^{\prime \prime}$, which coincides with a faint galaxy, they would form a wide-angle-tailed (WAT) radio source of about 1.3 Mpc in size (assuming it is a cluster radio galaxy). This seems unlikely, since the size would be quite large for a WAT (e.g., O'Donoghue et al. 1990) and since WAT sources are usually located at the cluster center. Although the above arguments may be weak, we suggest the reasonable possibility that at least the source D3 is a diffuse peripheral relic, similar to the source $1253+275$ in the Coma cluster (Giovannini et al. 1991), while the source D4 could be connected either to the mentioned point source or to the relic. More observations are necessary to investigate this point.

We obtained a $20 \mathrm{~cm}$ image of the radio halo after subtraction of the discrete unrelated sources. To this aim, we produced an image of discrete sources, by selecting from the original data set only the data from the long baselines, which do not contain the extended halo emission. This image was then subtracted from the image of Fig. 1. The halo map obtained in this way is presented in Fig. 3. The diffuse emission surrounding the halo is due to the 


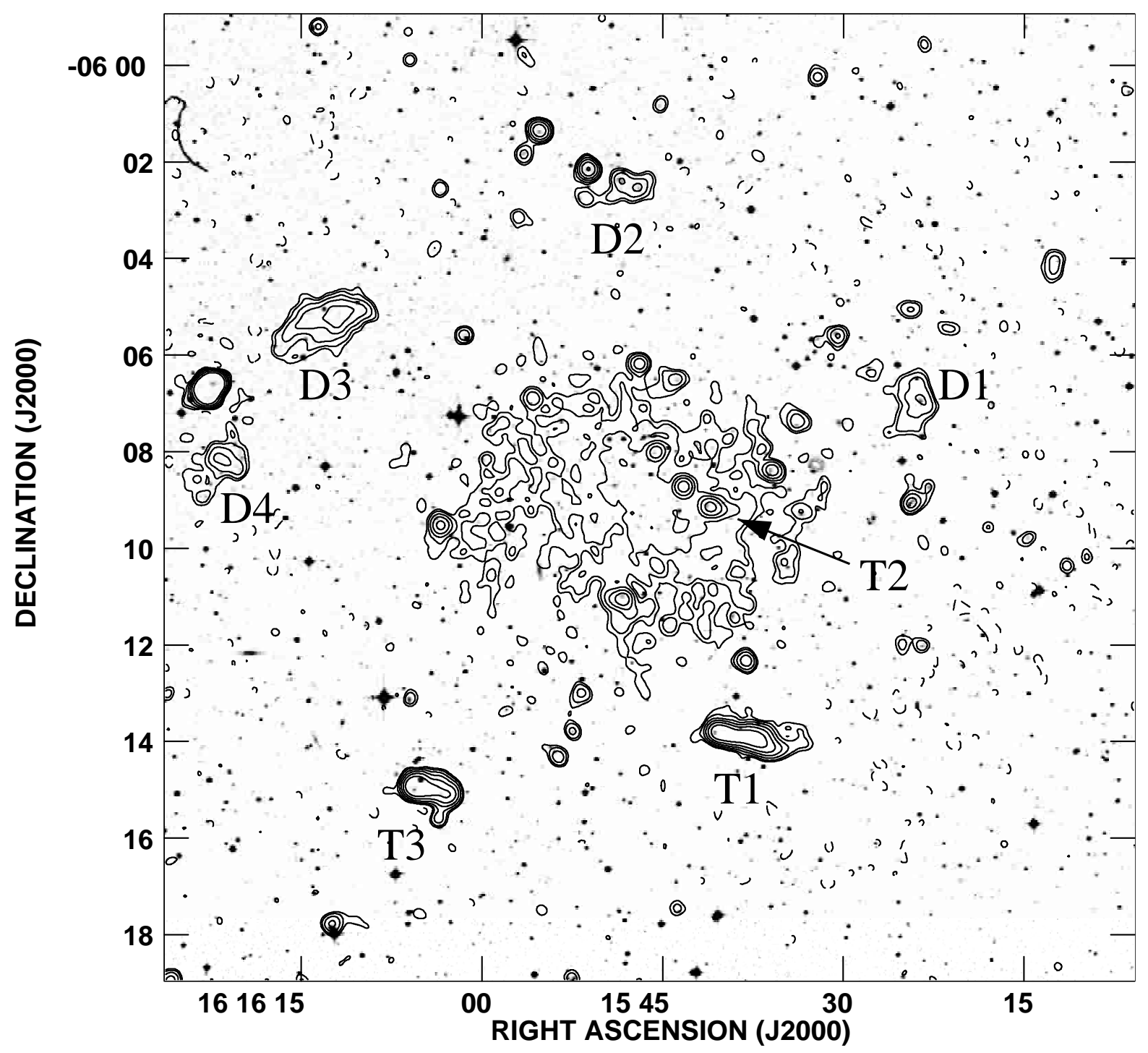

Fig. 2. Radio map at $20 \mathrm{~cm}$ with resolution of $15^{\prime \prime}$, overlayed onto the grey-scale image from the digitized Palomar Sky Survey. The $\sigma$ noise level in this map is $0.03 \mathrm{mJy} /$ beam. Contours are at $-0.09,0.09,0.15,0.3,0.6,1.2,2.5 \mathrm{mJy} /$ beam. Labels indicate the tailed radio galaxies and the diffuse features (see text).

relic and to the other diffuse features, which are extended and therefore difficult to be subtracted entirely. The parameters for the central radio halo, after subtraction of the unrelated sources, and for the relic are presented in Table 2 .

At $6 \mathrm{~cm}$, only the strongest discrete sources within and around the radio halo are well detected. The radio halo is not detected because of missing short spacings. Also the other diffuse features are completely resolved at $6 \mathrm{~cm}$.

Since the radio halo is not detected at $6 \mathrm{~cm}$, we attempted an estimate of its spectral index by comparing the images obtained from the two separate VLA frequencies at $20 \mathrm{~cm}$. Although the frequencies are quite close, the flux densities are slightly different, and the estimated spectral index $\left(S_{\nu} \propto \nu^{-\alpha}\right)$ is $\sim 1.6 \pm 0.3$. This steep spectrum is consistent with the values obtained in halo sources (e.g. Feretti \& Giovannini 1996). The map of the spectral index is quite noisy, so it is impossible to reveal any trend across the radio halo.

\subsection{Tailed radio sources}

Three radio sources in this cluster are found to show a tailed structure in the high resolution images. They are labeled T1, T2 and T3 in Fig. 2, and are listed in Table 3. They are all coincident with faint galaxies on the digitized Palomar Sky Survey images. Their radio images at high resolution are presented in Figs. 4-6, where a cross indicates the probable host galaxy. The total flux density and size given in Table 3 are derived from the maps at lower resolution, since some structure is missing in the high resolution images. The $6 \mathrm{~cm}$ images show the radio nucleus and only the beginning of the tail, confirming the tailed structure of these radiosources. It is worth noting that all 
Table 3. Properties of the tailed radio sources.

\begin{tabular}{|c|c|c|c|c|c|c|c|c|}
\hline Radiogal. & Label & $\begin{array}{l}\mathrm{RA}(\mathrm{J} 2 \\
\mathrm{h} \quad \mathrm{m}\end{array}$ & $\begin{array}{l}000) \\
\mathrm{s}\end{array}$ & $\begin{array}{l}\text { DEC } \\
\circ,\end{array}$ & 11 & & $\begin{array}{l}S_{20} \\
\text { mJy }\end{array}$ & $\begin{array}{l}\text { LLS } \\
\mathrm{kpc}\end{array}$ \\
\hline J1615-062 & $\mathrm{T} 1$ & $16 \quad 15$ & 40.6 & -06 & 13 & 48 & $34.5 \pm 0.5$ & 630 \\
\hline J1615-061 & $\mathrm{T} 2$ & $16 \quad 15$ & 41.3 & -06 & 09 & 08 & $6.0 \pm 0.3$ & 170 \\
\hline J1616-062 & $\mathrm{T} 3$ & $16 \quad 16$ & 05.6 & -06 & 14 & 56 & $24.9 \pm 0.4$ & 380 \\
\hline
\end{tabular}

Column 1: name, Col. 2: label according to Fig. 2; Col. 3: position of the radio nucleus; Col. 4: flux density at $20 \mathrm{~cm}$ derived from the image at the lowest resolution; Col. 5: largest linear size obtained from the image at the lowest resolution.

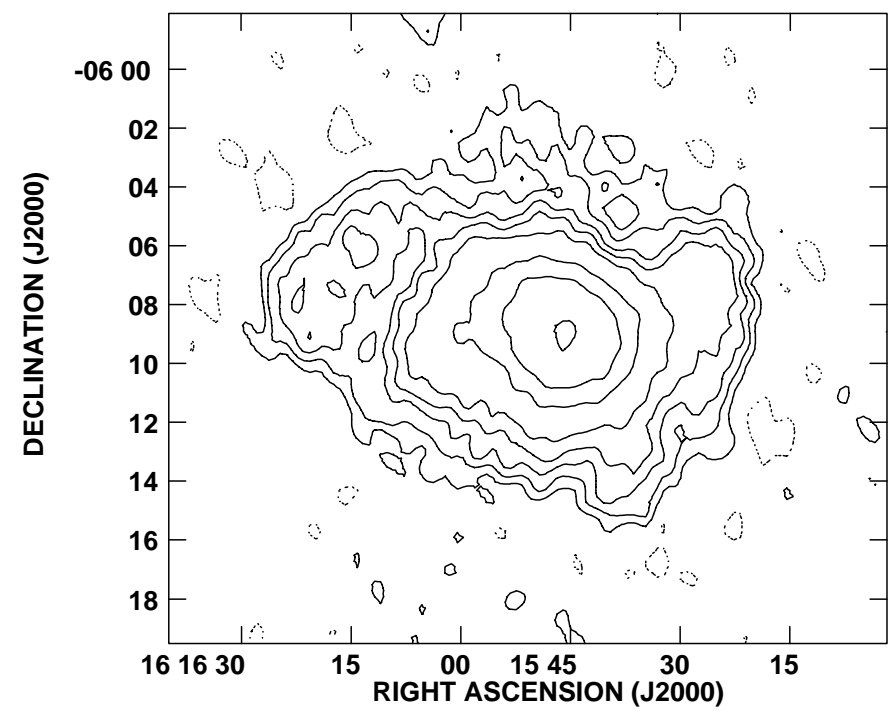

Fig. 3. Radio map at $20 \mathrm{~cm}$ of the diffuse emission, after subtraction of the discrete unrelated sources. The resolution is the same as in Fig. 1, whereas the noise level is $0.05 \mathrm{mJy} /$ beam. Contour levels are $-0.15,0.15,0.3,0.5,0.7$, 1, 2, 3, $5 \mathrm{mJy} /$ beam.

3 radio galaxies have the tails oriented in the same direction (West). This could be explained by the presence of merger-induced bulk motion of the intergalactic medium, as suggested by Bliton et al. (1998).

\section{Radio versus $\mathrm{X}$-ray comparison}

In Fig. 7 we show the overlay of the radio and X-ray images, for a morphological comparison. The radio image is the same as that presented in Fig. 1. The X-ray image was obtained with the ROSAT Position Sensitive Proportional Counter (PSPC) with a total exposure time of $12133 \mathrm{~s}$ (Elbaz et al. 1995). The image presented here has been reprocessed with a more accurate determination of the exposure map, and refers to the $0.5-2 \mathrm{keV}$ energy range, in order to have a lower background. It has been corrected for the vignetting effect, and smoothed with a Gaussian of $\sigma=30^{\prime \prime}$. As noted by Elbaz et al. (1995), the cluster $\mathrm{X}$-ray structure is clearly non spherical and in particular the shape is elongated in the E-W direction. There is also some evidence that the axis of the elliptically shaped distribution in the central region of the cluster has a
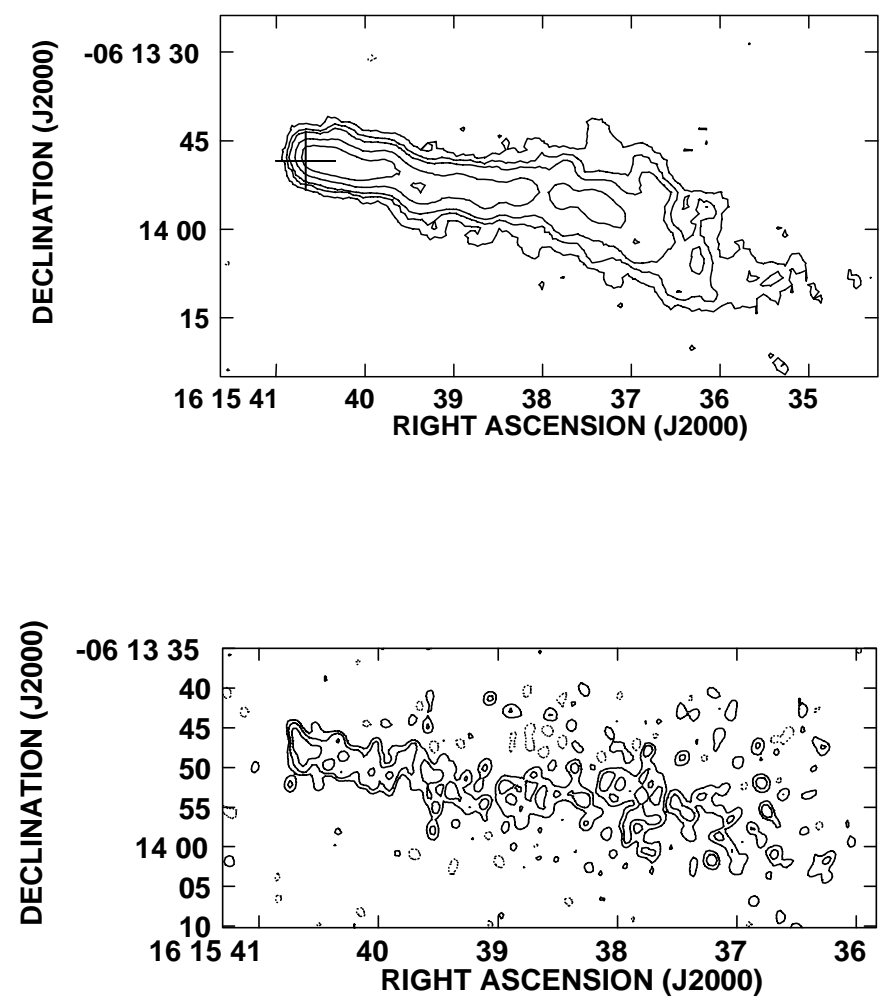

Fig. 4. High resolution radio images at $20 \mathrm{~cm}$ of the tailed radio galaxy J1615-062 (T1). The upper panel shows the map with resolution of $4^{\prime \prime}$ and $\sigma$ noise level of $0.035 \mathrm{mJy} /$ beam. Contours are at $-0.1,0.1,0.2,0.3,0.6,1 \mathrm{mJy} /$ beam. The cross indicates the position of a faint galaxy. The lower panel shows the image with the highest resolution, of $1.7^{\prime \prime} \times 1.4^{\prime \prime}\left(@ \mathrm{PA}=-19^{\circ}\right)$. The $\sigma$ noise level is $0.018 \mathrm{mJy} /$ beam. Contours are at $-0.06,0.06$, $0.12,0.25 \mathrm{mJy} /$ beam.

different orientation, i.e. inclined between the E-W and the NE-SW direction. These morphological features are strickingly similar to those found in the radio structure, confirming the connection between hot and relativistic plasma, found in other clusters (Deiss et al. 1997; Feretti 1999; Liang et al. 2000). In addition, we note that there appears to be a compact X-ray source at the location of the radio source D2.

We have performed a quantitative point-to-point comparison of the radio and X-ray brightness, following the same approach used by Govoni et al. (2001) for the analysis of the clusters Coma, A2255, A2319, A2744. The radio 


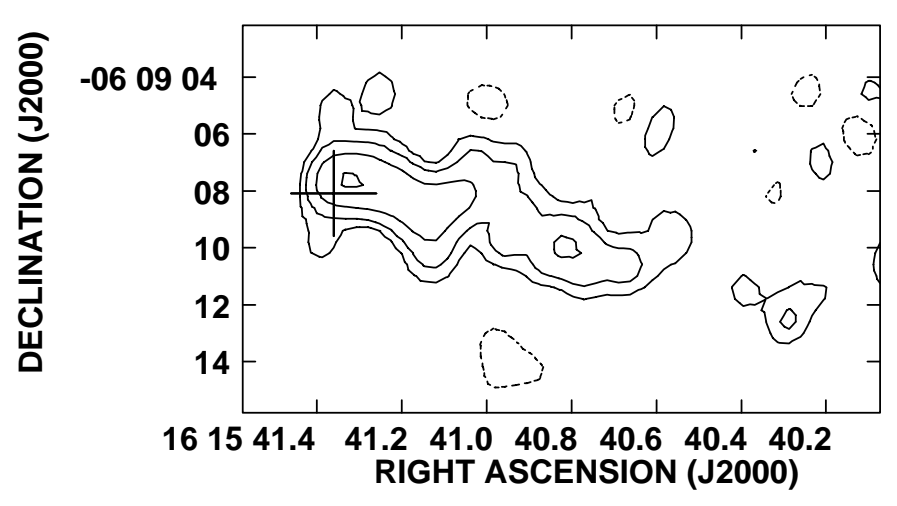

Fig. 5. Radio map at $20 \mathrm{~cm}$ of the radio galaxy J1615-061 (T2) with resolution of $1.7^{\prime \prime} \times 1.4^{\prime \prime}\left(@ \mathrm{PA}=-19^{\circ}\right)$. The $\sigma$ noise level in this map is $0.018 \mathrm{mJy} /$ beam. Contours are at $-0.06,0.06$, $0.12,0.25,0.5 \mathrm{mJy} / \mathrm{beam}$. The cross indicates the position of a faint galaxy.

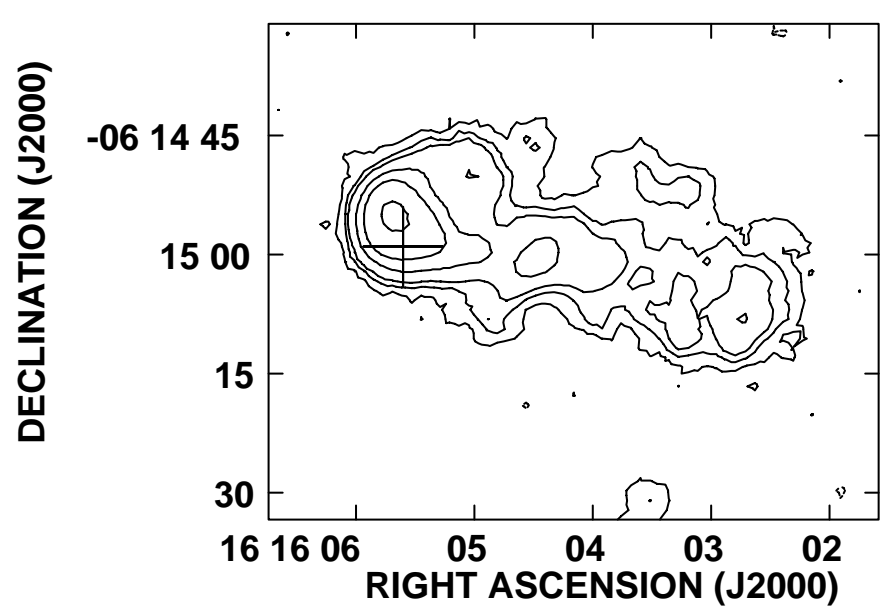

Fig. 6. Radio map at $20 \mathrm{~cm}$ of the tailed radio galaxy J1616062 (T3) with resolution of $4^{\prime \prime}$. The $\sigma$ noise level in this map is $0.035 \mathrm{mJy} /$ beam. Contours are at $-0.1,0.1,0.2,0.3,0.6,1$, $2 \mathrm{mJy} /$ beam. The cross indicates the position of a faint galaxy.

and X-ray images are suitable for such a study, having similar resolutions. In the X-ray map the constant background was subtracted. The image obtained after subtraction of discrete sources and corrected for the primary beam attenuation was used as a radio map. The plot of the radio versus the X-ray brightness is shown in Fig. 8. The close similarity between radio and X-ray structures is demonstrated by the correlation between these two parameters: a higher X-ray brightness is associated with a higher radio brightness. The data was fitted to a power law relation of the type:

$B_{\text {radio }}=a B_{\mathrm{X}-\text { ray }}^{\mathrm{b}}$.

With the radio brightness $B_{\text {radio in }} \mathrm{mJy} \operatorname{arcsec}^{-2}$ and

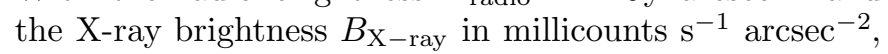
the parameters of the best fit are $b=0.64 \pm 0.05$ and $a=0.03 \pm 0.01$. Therefore the correlation indicates that the radial decline of the non-thermal radio component is slower that that of the thermal one. Relations with $b<1$ were also found in the clusters Coma and A2319, whereas

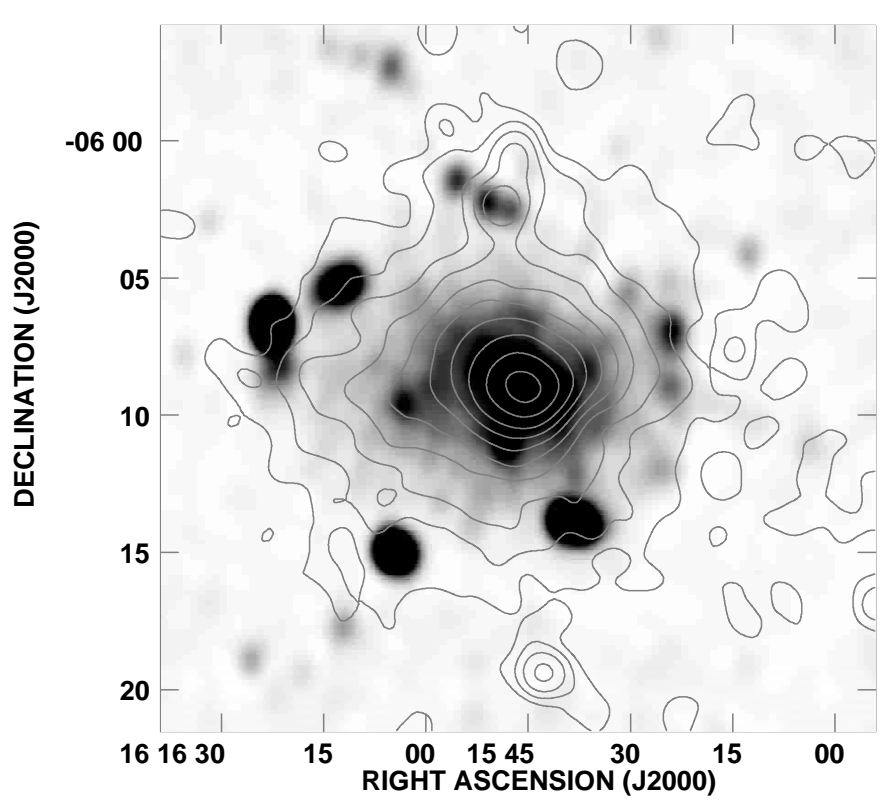

Fig. 7. Overlay of the radio map (grey scale) onto the X-ray image (contours). The contour levels are at $0.045,0.07,0.11$, $0.18,0.28,0.45,0.71,1.1,1.8,2.8$ millicts s ${ }^{-1}$ pixel $^{-1}$, with a pixel size of $15^{\prime \prime} \times 15^{\prime \prime}$.

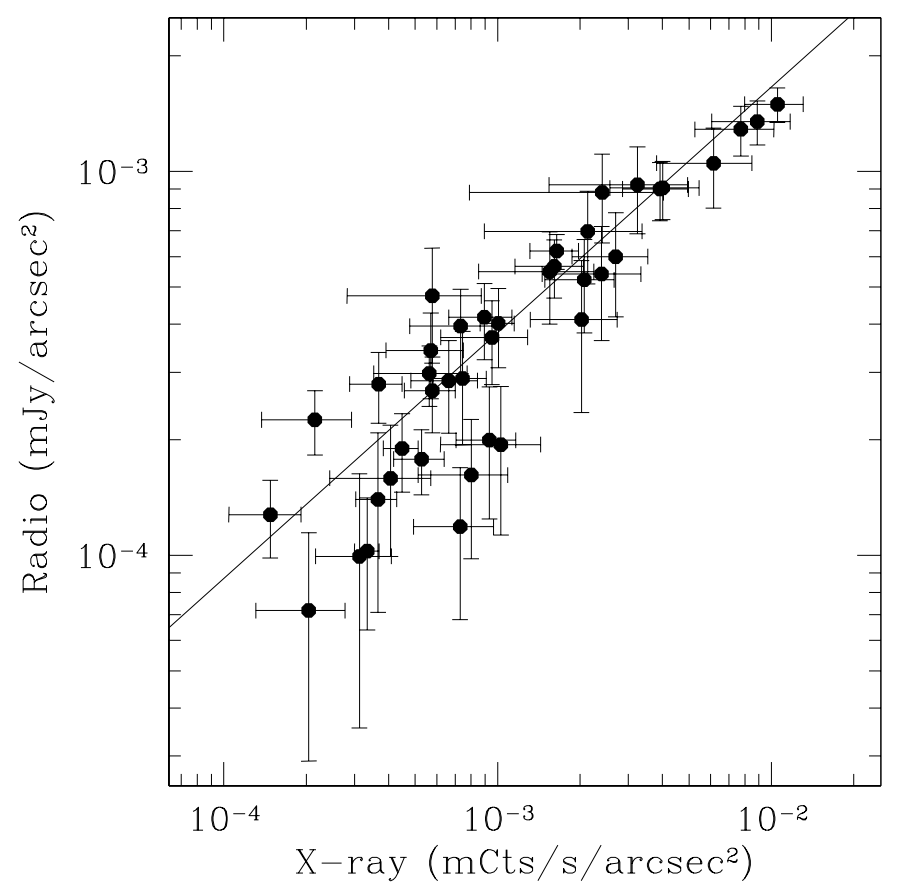

Fig. 8. Plot of the radio brightness versus the X-ray brightness, obtained in cells of $90^{\prime \prime}$ size. The errors are represented by the rms in each cell. The best fit is indicated by a continuous line.

the correlation between the radio and the X-ray brightness was found to be linear in A2255 and A2744 $(b \sim 1$, Govoni et al. 2001).

\section{BeppoSAX X-ray observation}

The cluster was observed in February 98 for a total exposure time of $109 \mathrm{ksec}$. Spatially resolved spectroscopy 
derived from the data obtained with the Medium-Energy Concentrator Sectrometer (MECS) has been published by Irwin \& Bregman (2000). The average temperature between 1.65 and $10.5 \mathrm{keV}$ is found to be $11 \pm 0.6 \mathrm{keV}$. The azimuthally averaged radial temperature profile shows a rather constant trend up to a distance of $10^{\prime}$ from the cluster center. This result agrees with the ASCA result of White (2000) but disagrees strongly with the result from ASCA and ROSAT by Markevitch et al. (1996), who found a temperature decrease at large distance.

We have analysed the data from the high energy detector PDS, operating in the $15-200 \mathrm{keV}$ energy range, with a field of view of $1.3^{\circ}$ (FWHM, hexagonal) to search for hard X-ray emission, as detected in the Coma cluster (Fusco-Femiano et al. 1999; Rephaeli et al. 1999) and A2256 (Fusco-Femiano et al. 2000) which both show extended radio regions. The PDS instrument uses the rocking collimator technique for background subtraction. The background level is the lowest obtained thus far with highenergy instruments on board satellites and it is very stable, thanks to the equatorial orbit. No modeling of the time variation of the background is required (see FuscoFemiano et al. 1999).

The data analysis gives evidence for hard X-ray emission at a confidence level of $\sim 3.4 \sigma$, with a count rate of $0.219 \pm 0.065 \mathrm{c} \mathrm{s}^{-1}$ in the PDS energy range. The data is well fitted by a thermal component at the average cluster temperature of $11 \mathrm{keV}$. For the non-thermal emission, an X-ray flux upper limit of $\sim 5.6 \times 10^{-12} \mathrm{erg} \mathrm{cm}^{-2} \mathrm{~s}^{-1}$ in the $20-80 \mathrm{keV}$ energy range is derived. This upper limit is about 4 and 2 times lower than the non-thermal flux measurements obtained in the same energy range for Coma (Fusco-Femiano et al. 1999) and A2256 (Fusco-Femiano et al. 2000), respectively.

\section{Discussion}

\subsection{Halo origin}

The diffuse halo in A2163 is more powerful and more extended than the prototype halo source in the Coma cluster, Coma C. Like Coma C, it is rather regular in shape. The properties of radio halos and relics in clusters have been recently reviewed by Feretti (2000) and Giovannini et al. (2000). The formation of radio halos has been found to be strictly related to the X-ray properties of the host clusters and to the presence of cluster merger processes. In particular, the percentage of clusters showing radio halos is much higher in a sample of X-ray luminous clusters $\left(\sim 30 \%\right.$ for $\left.L_{\mathrm{X}-\text { ROSAT }}>10^{45} \mathrm{erg} \mathrm{s}^{-1}\right)$ than in a complete cluster sample $(\sim 5 \%)$. The clusters hosting a diffuse source have a significantly higher X-ray luminosity than clusters without a diffuse source. Moreover, the radio properties of halos are linked to the properties of the X-ray emitting gas through correlations (Feretti 2000; Liang et al. 2000): the halo monochromatic radio power correlates with the X-ray luminosity, the X-ray temperature and the cluster mass. A correlation is also found between the largest radio size of halos and relics and the cluster X-ray luminosity, with more X-ray luminous clusters hosting larger sources.

The properties of the radio halo in A2163 are fully consistent with the above suggestions. Indeed, this cluster is very hot and massive, and hosts the most powerful and extended halo known. From the temperature maps obtained with ASCA and recently with Chandra et al. (1996) and Markevitch et al. (2000) inferred that the cluster inner region is in a highly nonrelaxed state, with shocks or streams of shock-heated gas, probably due to a recent merger. This is also confirmed by Squires et al. (1997) from the study of the distribution of cluster light, thermal gas and dark matter. The merger process in this cluster is likely to provide the energy necessary for particle reacceleration, according to a model suggested by Brunetti et al. (2001) for the Coma cluster.

The radio structure of this halo shows close similarity to the structure of the X-ray emitting gas. This is evident from the overlay between radio and X-ray images, and from the correlation between radio and X-ray brightness. The connection between the hot and relativistic plasma could still reflect the connection between the maintenance of the radio halo and the energy provided by the cluster merger, and, when available for a large sample of clusters, could be used to discriminate between models of halo formation (see Govoni et al. 2001).

The presence of a relic in this cluster is very interesting. It is located at about $2.2 \mathrm{Mpc}$ from the cluster center and is oriented roughly perpendicular to the cluster radial direction, like the other well know relics. The particles radiating in the relic could be reaccelerated in a shock produced by the cluster merger, as in A3667 (Roettiger et al. 1999). Unlike the cluster A3667, which has no radio halo at the center, the existence in the same cluster of both a central radio halo and a peripheral relic is not uncommon (Feretti 1999). This points in favor of a common origin for halos and relics, which could be investigated when detailed data on the cluster merger state and evolution will be available from the new generation X-ray telescopes.

\subsection{Inverse Compton emission}

Non-thermal hard X-ray radiation is expected in galaxy clusters with radio halos, because of inverse Compton (IC) scattering by the radio emitting relativistic electrons with the CMB photons. The alternative interpretations to the IC model proposed in the literature for the non-thermal emission detected in Coma (Enßlin et al. 1999; Sarazin \& Kempner 2000; Blasi \& Colafrancesco 1999; Dogiel 2000; Blasi 2000) show serious difficulties, as recently pointed out by Petrosian (2001).

From the PDS data analysis we derive evidence for hard X-ray emission at a confidence level of $\sim 3.4 \sigma$. This can be accounted for by the cluster thermal emission, whereas for the non-thermal emission only an upper limit is derived. The detection of non-thermal emission in A2163 
appears problematic for the great distance and high gas temperature of this cluster.

Within the framework of the IC model, it is possible to derive, only using observables (see Rephaeli 1979), a lower limit to the volume-averaged intracluster magnetic field combining the synchrotron radio flux with the X-ray flux upper limit for the non-thermal component (see Sect. 5). Using the radio data reported in Table 2 for the halo, we obtain $B \gtrsim 0.28 \mu \mathrm{G}$. Assuming a radius of $\sim 1.4 \mathrm{Mpc}$ for the radio emitting region, at the distance of the cluster, we derive an upper limit to the electron energy density, $\rho_{\mathrm{e}}$, of $\sim 6.5 \times 10^{-14} \mathrm{erg} \mathrm{cm}^{-3}$. The figures obtained in this way are consistent with the equipartition values (see Table 2; note that the minimum energy density in the table includes the contributions of both the radiating particles and the magnetic field). The derived limits are not sufficient to establish whether the radio halo is in equipartition conditions or not.

Acknowledgements. We are indebted to Monique Arnaud for providing the X-ray ROSAT image, and for helpful discussions. The point-to-point radio-X-ray comparison was performed with the Synage++ package developed by Matteo Murgia. We thank Barry O'Connell for comments on the manuscript. The National Radio Astronomy Observatory is operated by Associated Universities, Inc., under contract with the National Science Foundation.

\section{References}

Arnaud, M., Hughes, J. P., Forman, W., et al. 1992, ApJ, 390, 345

Blasi, P. 2000, ApJ, 532, L9

Blasi, P., \& Colafrancesco, S. 1999, Astropart. Phys., 12, 169

Bliton, M., Rizza, E., Burns, J. O., Owen, F. N., \& Ledlow, M. J. 1998, MNRAS, 301, 609

Brunetti, G., Setti, G., Feretti, L., \& Giovannini, G. 2001, MNRAS, 320, 365

Burns, J. O., Rhee, G., Owen, F. N., \& Pinkey, J. 1994, ApJ, 423,94

Désert, F.-X., Benoit, A., Gaertner, S., et al. 1998, NewA, 655, 669

Deiss, B. M., Reich, W., Lesch, H., \& Wielebinski, R. 1997, A\&A, 321, 55

Dogiel, V. A. 2000, A\&A, 357, 66

Elbaz, D., Arnaud, M., \& Böhringer, H. 1995, A\&A, 293, 337
Enßlin, T. A., Lieu, R., \& Biermann, P. L. 1999, A\&A, 344, 409

Feretti, L. 1999, in Diffuse Thermal and Relativistic Plasma in Galaxy Clusters, ed. H. Böhringer, L. Feretti, \& P. Schuecker, MPE Report, No. 271 (MPE Garching), 3

Feretti, L. 2000, in The Universe at low Radio Frequencies, Symp. IAU 199, Pune (India), in press [astro-ph/0006379]

Feretti, L., \& Giovannini, G. 1996, in Extragalactic Radio Sources, IAU Symp. 175, ed. R. Ekers, C. Fanti, \& L. Padrielli (Kluwer Academic Publisher), 333

Fusco-Femiano, R., Dal Fiume, D., Feretti, L., et al. 1999, ApJ, 513, L21

Fusco-Femiano, R., Dal Fiume, D., De Grandi, S., et al. 2000, ApJ, 534, L10

Giovannini, G., Feretti, L., \& Stanghellini, C. 1991, A\&A, 252, 528

Giovannini, G., Feretti, L., Venturi, T., Kim, K.-T., \& Kronberg, P. P. 1993, ApJ, 406, 399

Giovannini, G., Feretti, L., \& Govoni, F. 2000, in The Universe at low Radio Frequencies, Symp. IAU 199, Pune (India), in press [astro-ph/0006380]

Govoni, F., Enßlin, T. A., Feretti, L., \& Giovannini, G. 2001, A\&A, in press [astro-ph/0101418]

Henry, J. P., \& Briel, U. G. 1993, Adv. Space Res., 13(12), 191

Herbig, T., \& Birkinshaw, M. 1994, BAAS, 26(4), 1403

Holzapfel, W. L., Ade, P. A. R., Church, S. E., et al. 1997, ApJ, 481, 35

Irwin, J. A., \& Bregman, J. N. 2000, ApJ, 538, 554

Liang, H., Hunstead, R. W., Birkinshaw, M., \& Andreani, P. 2000, ApJ, 544, 686

Markevitch, M., Mushotzky, R., Inoue, H., et al. 1996, ApJ, 456, 437

Markevitch, M., Vikhlinin, A., Mazzotta, P., \& VanSpeybroeck, L. 2000, in X-ray astronomy 2000 , ed. R. Giacconi, L. Stella, \& S. Serio, ASP Conf. Ser., in press [astro-ph/0012215]

O’Donoghue, A. A., Owen, F. N., \& Eilek, J. A. 1990, ApJS, 72, 75

Petrosian, V. 2001, ApJ, in press [astro-ph/0101145]

Rephaeli, Y. 1979, ApJ, 227, 364

Rephaeli, Y., Gruber, D., \& Blanco, P. 1999, ApJ, 511, 21

Roettiger, K., Stone, J. M., \& Burns, J. O. 1999, ApJ, 518, 594

Sarazin, C. L., \& Kempner, J. C. 2000, ApJ, 533, 73

Squires, G., Neumann, D. M., Kaiser, N., et al. 1997, ApJ, 482, 648

Struble, M. F., \& Rood, H. J. 1999, ApJS, 125, 35

White, D. A. 2000, MNRAS, 312, 649 